\title{
Parameter degeneracy and hierarchy sensitivity of NOvA in presence of sterile neutrino
}

\section{Monojit Ghosh*}

Department of Physics, Tokyo Metropolitan University, Hachioji, Tokyo 192-0397, Japan

E-mail: mghosh@phys.se.tmu.ac.jp

\section{Shivani Gupta}

Center of Excellence for Particle Physics at the Terascale (CoEPP), University of Adelaide, Adelaide SA 5005, Australia

E-mail: shivani.guptaeadelaide.edu.au

\section{Zachary M. Matthews}

Center of Excellence for Particle Physics at the Terascale (CoEPP), University of Adelaide, Adelaide SA 5005, Australia

E-mail: zachary.matthews@adelaide.edu.au

\section{Pankaj Sharma}

Center of Excellence for Particle Physics at the Terascale (CoEPP), University of Adelaide, Adelaide SA 5005, Australia

E-mail: pankaj.sharmaeadelaide.edu.au

\section{Anthony G. Williams}

Center of Excellence for Particle Physics at the Terascale (CoEPP), University of Adelaide, Adelaide SA 5005, Australia

E-mail: anthony.williamseadelaide.edu.au

The first hint of neutrino mass hierarchy is expected to come from the NOvA experiment in Fermilab as the present best-fit parameter space i.e., normal hierarchy and $\delta_{C P}=-90^{\circ}$ is the favourable parameter space for NOvA where there is no degeneracy. But this situation may change if the standard three flavour framework is not complete and there is existence of new physics. In this work we consider the presence of an extra light sterile neutrino at the eV scale and study the new degeneracies which are absent in the standard three flavour framework. We also study the effect of these new degeneracies on the hierarchy measurement of NOvA.

The 19th International Workshop on Neutrinos from Accelerators-NUFACT2017

25-30 September, 2017

Uppsala University, Uppsala, Sweden

\footnotetext{
*Speaker.
} 


\section{Introduction}

In the standard three flavour framework, neutrino oscillation in which neutrinos change their flavour is described by six parameters: three mixing angles: $\theta_{12}, \theta_{13}, \theta_{23}$, two mass squared differences: $\Delta_{21}\left(m_{2}^{2}-m_{1}^{2}\right)$, and $\Delta_{31}\left(m_{3}^{2}-m_{1}^{2}\right)$ and one phase $\delta_{13}$. Among them one of the major unknown is the sign of $\Delta_{31}$ or the neutrino mass hierarchy. It can be either normal i.e., $\Delta_{31}>0$ $(\mathrm{NH})$ or inverted i.e., $\Delta_{31}<0(\mathrm{IH})$. It is well known that for the favourable parameter space where there is no degeneracy, then NOvA [1] can determine neutrino mass hierarchy at more that $2 \sigma$ C.L. The global analysis of the world neutrino data suggests that the best fit parameter space i.e., $\mathrm{NH}$ with $\delta_{13}=-90^{\circ}[2,3,4]$ is indeed the favourable parameter space for NOvA and thus it is expected that the first hint of neutrino mass hierarchy will come from the NOvA experiment. But the situation can be different if there exists new physics. In presence of new physics there can be additional degeneracies which can spoil the hierarchy sensitivity of NOvA even for the favourable parameter space. In this work we consider the existence of an extra light sterile neutrino at the $\mathrm{eV}$ scale [5] i.e. the $3+1$ scenario. In this present work our aim is to identify the new degeneracies and study their effect in the determination of hierarchy in NOvA.

\section{Oscillation parameters in $3+1$ scheme}

In presence of one extra light sterile neutrino, we parametrize the PMNS matrix as

\begin{tabular}{|c|c|c|}
\hline $4 v$ Parameters & True Value & Test Value Range \\
\hline $\sin ^{2} \theta_{12}$ & 0.304 & N/A \\
$\sin ^{2} 2 \theta_{13}$ & 0.085 & N/A \\
$\theta_{23}^{\mathrm{LO}}$ & $40^{\circ}$ & $\left(40^{\circ}, 50^{\circ}\right)$ \\
$\theta_{23}^{\mathrm{HO}}$ & $50^{\circ}$ & $\left(40^{\circ}, 50^{\circ}\right)$ \\
$\sin ^{2} \theta_{14}$ & 0.025 & $\mathrm{~N} / \mathrm{A}$ \\
$\sin ^{2} \theta_{24}$ & 0.025 & $\mathrm{~N} / \mathrm{A}$ \\
$\theta_{34}$ & $0^{\circ}$ & $\mathrm{N} / \mathrm{A}$ \\
$\delta_{13}$ & $-90^{\circ}$ & $\left(-180^{\circ}, 180^{\circ}\right)$ \\
$\delta_{14}$ & $-90^{\circ}, 0^{\circ}, 90^{\circ}$ & $\left(-180^{\circ}, 180^{\circ}\right)$ \\
$\delta_{34}$ & $0^{\circ}$ & $\mathrm{N} / \mathrm{A}$ \\
$\Delta_{21}$ & $7.5 \times 10^{-5} \mathrm{eV}^{2}$ & $\mathrm{~N} / \mathrm{A}$ \\
$\Delta_{31}$ & $2.475 \times 10^{-3} \mathrm{eV}^{2}$ & $(2.2,2.6) \times 10^{-3} \mathrm{eV}^{2}$ \\
$\Delta_{41}$ & $1 \mathrm{eV}^{2}$ & $\mathrm{~N} / \mathrm{A}$ \\
\hline
\end{tabular}

Table 1: Expanded $4 v$ parameter true values and test marginalisation ranges, parameters with N/A are not marginalised over.

$$
U_{\mathrm{PMNS}}^{4 v}=U\left(\theta_{34}, \delta_{34}\right) U\left(\theta_{24}, 0\right) U\left(\theta_{14}, \delta_{14}\right) U_{\mathrm{PMNS}}^{3 v}
$$

where

$$
U_{\mathrm{PMNS}}^{3 v}=U\left(\theta_{23}, 0\right) U\left(\theta_{13}, \delta_{13}\right) U\left(\theta_{12}, 0\right) .
$$


where $U\left(\theta_{i j}, \delta_{i j}\right)$ contains a corresponding $2 \times 2$ mixing matrix:

$$
U^{2 \times 2}\left(\theta_{i j}, \delta_{i j}\right)=\left(\begin{array}{cc}
\mathrm{c}_{i j} & \mathrm{~s}_{i j} e^{i \delta_{i j}} \\
-\mathrm{s}_{i j} e^{i \delta_{i j}} & \mathrm{c}_{i j}
\end{array}\right)
$$

embedded in an $n \times n$ array in the $i, j$ sub-block. Thus in this case the neutrino oscillation parameter space is increased by three more mixing angles: $\theta_{14}, \theta_{24}$ and $\theta_{34}$, two more Dirac type CP phases i.e., $\delta_{14}$ and $\delta_{34}$ and one more mass squared difference: $\Delta_{41}\left(m_{4}^{2}-m_{1}^{2}\right)$. In $3+1$ case, the appearance channel expression in vacuum is given by [6]

$$
P_{\mu e} \simeq 4 s_{23}^{2} s_{13}^{2} \sin ^{2} \Delta+8 s_{13} s_{12} c_{12} s_{23} c_{23}(\alpha \Delta) \sin \Delta \cos \left(\Delta \pm \delta_{13}\right)+4 s_{14} s_{24} s_{13} s_{23} \sin \Delta \sin \left(\Delta \pm \delta_{13} \mp \delta_{14}\right)
$$

where $\Delta \equiv \Delta_{31} L / 4 E, \alpha \equiv \Delta_{21} / \Delta_{31}$ with $L$ being the baseline and $E$ is the energy. For our present work we list our choice of parameters in Table 1 [7].
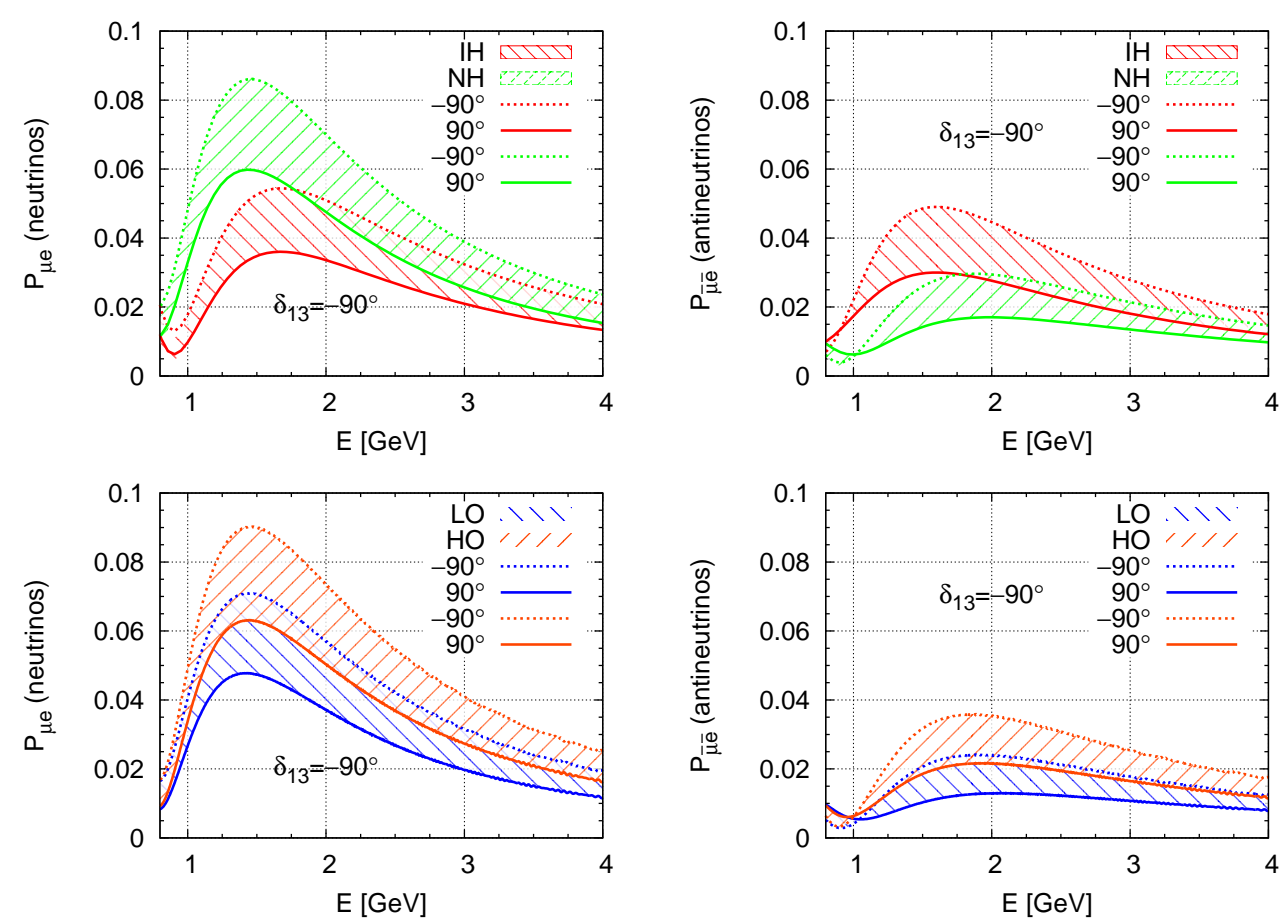

Figure 1: $v_{\mu} \rightarrow v_{e}$ oscillation probability bands for $\delta_{13}=-90^{\circ}$. Left panels are for neutrinos and right panels are for antineutrinos. The upper panel shows the hierarchy- $\delta_{14}$ degeneracy and the lower panels shows the octant- $\delta_{14}$ degeneracy.

In the table, LO implies the lower octant of $\theta_{23}$ and $\mathrm{HO}$ implies higher octant of $\theta_{23}$. We have generated all our results with the GLoBES software [8].

\section{Degeneracy at the probability level}

In fig. 1 , we have plotted the appearance probability vs energy for $\delta_{13}=-90^{\circ}$ and the bands are due to variation of $\delta_{14}$. The upper panels show the hierarchy- $\delta_{14}$ degeneracy and the lower 

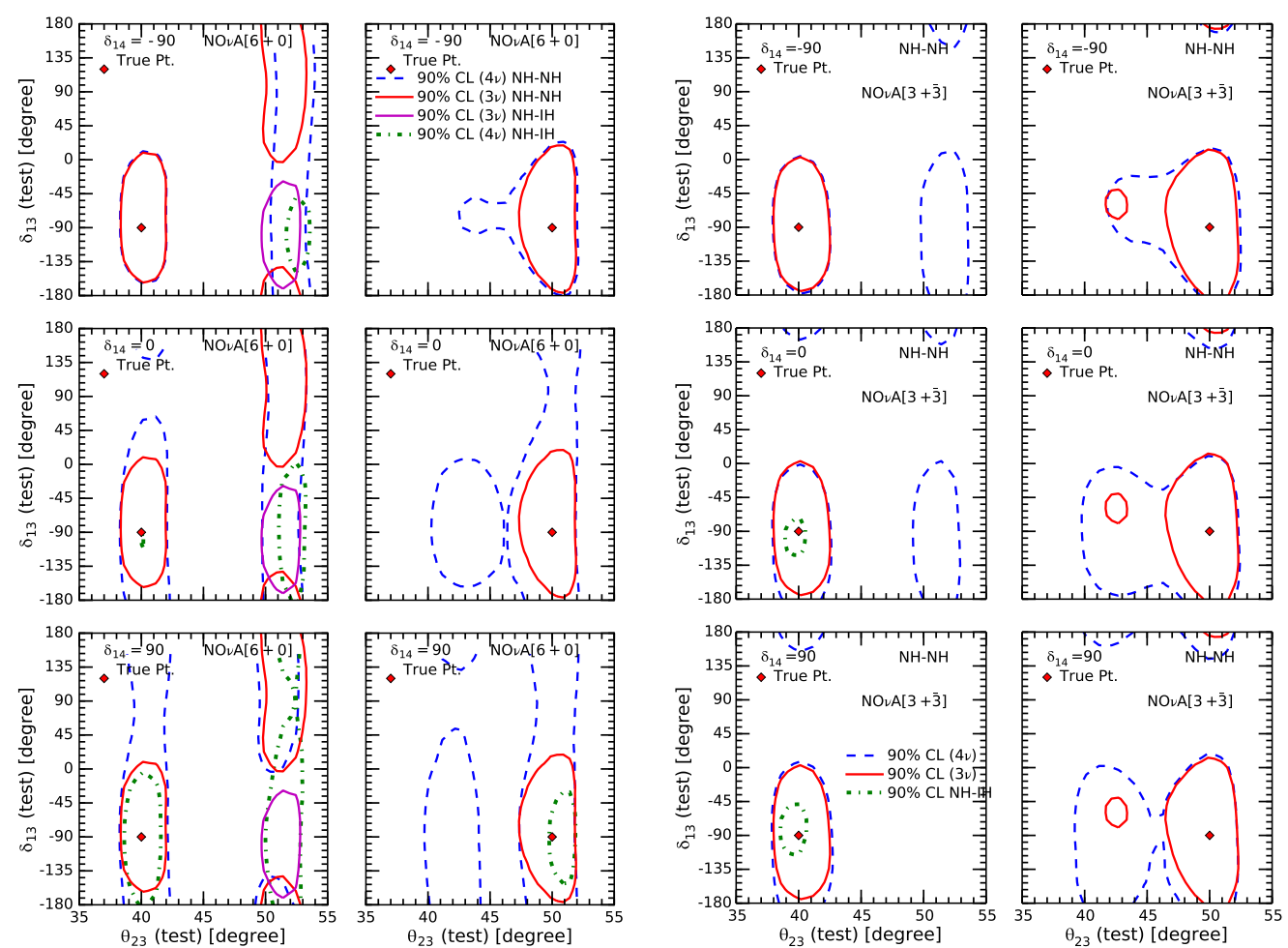

Figure 2: Contour plots in the $\theta_{23}$ (test) vs $\delta_{13}$ (test) plane for two different true values of $\theta_{23}=40^{\circ}$ (first and third column) and $50^{\circ}$ (second and fourth column) for NOvA $(6+\overline{0})$ (first and second column) and $(3+\overline{3})$ (third and fourth column). The first, second and third rows are for $\delta_{14}=-90^{\circ}, 0^{\circ}$ and $90^{\circ}$ respectively. The true value for the $\delta_{13}$ is taken to be $-90^{\circ}$. The true hierarchy is $\mathrm{NH}$. We marginalize over the test values of $\delta_{14}$. Also shown is the contours for the $3 v$ flavor scenario.

panels depict octant- $\delta_{14}$ degeneracy. From the upper panels we see that we have degeneracies in $\left\{\mathrm{NH}, \delta_{14}=90^{\circ}\right\}$ with $\left\{\mathrm{IH}, \delta_{14}=-90^{\circ}\right\}$ for neutrinos and $\left\{\mathrm{NH}, \delta_{14}=-90^{\circ}\right\}$ with $\left\{\mathrm{IH}, \delta_{14}=90^{\circ}\right\}$ for antineutrinos. Thus we understand that this degeneracy can be removed with a balanced run of neutrinos and antineutrinos. From the lower panels we see that there is degeneracies in LOO, $\left.\delta_{14}=-90^{\circ}\right\}$ with $\left\{\mathrm{HO}, \delta_{14}=90^{\circ}\right\}$ for both neutrinos and antineutrinos. Thus it is clear that this degeneracy is unremovable. It was shown in Ref. [9] that due to this degeneracy, the octant determination of the long-baseline experiments is highly compromised.

\section{Degeneracies at the event level}

To Show the degeneracies at the event level, in Fig. 2 we have given the contour plots in the $\theta_{23}$ (test) - $\delta_{13}$ (test) plane. The true point is represented by the red diamond. In these panels, red and purple contours correspond to the right hierarchy and wrong hierarchy solutions respectively for the three generation case and the blue and green contours correspond to right hierarchy and wrong hierarchy solutions respectively for the $3+1$ case. By comparing the pure neutrino results of NOvA labeled as $\mathrm{NOvA}(6+0)$ and mixed neutrino-antineutrino results labeled as $\mathrm{NO} v \mathrm{~A}(3+3)$ we notice that for three generation case, the all the degenerate solutions are almost gone when antineutrino 

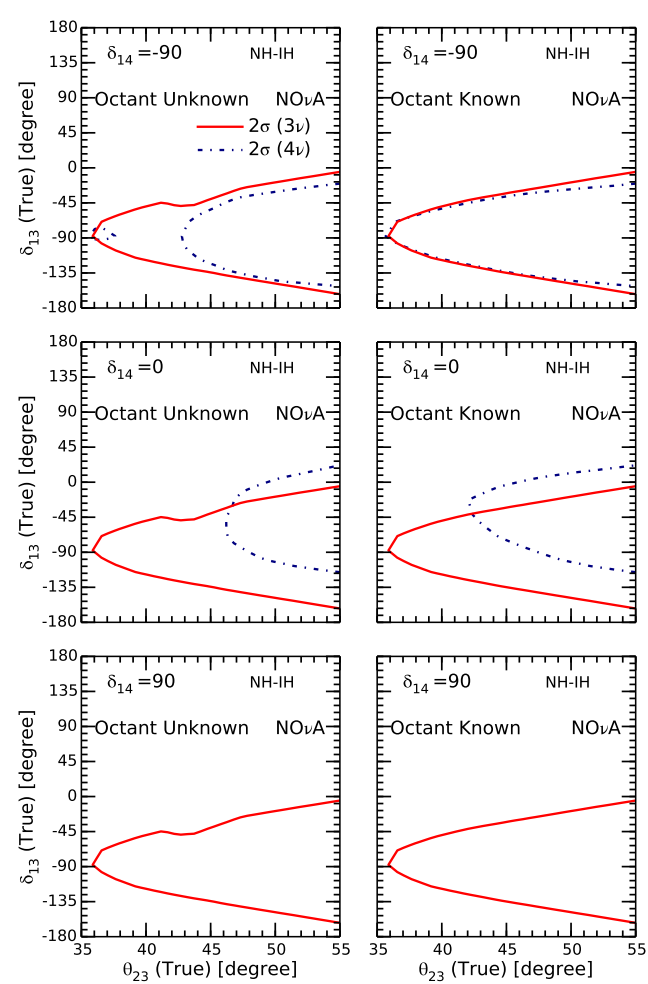

Figure 3: Contour plots at $2 \sigma$ C.L. in the $\theta_{23}$ (true) vs $\delta_{13}$ (true) plane for Octant Unknown (left panel) and Octant Known (right panel) scenarios for NOvA $(3+\overline{3})$. The first, second and third rows are for $\delta_{14}=-90^{\circ}$, $0^{\circ}$ and $90^{\circ}$ respectively. The true and test hierarchies are chosen to be normal $(\mathrm{NH})$ and inverted hierarchy (IH) respectively. Also shown contours for the $3 v$ flavor scenario.

data is considered. But for the $3+1$ case, we notice that the wrong hierarchy solutions are almost gone but the wrong octant solutions does not get removed.

Thus from the above discussion we understand that even for $\mathrm{NH}$ and $\delta_{13}=-90^{\circ}$, where there is almost no degeneracy in the three generation case, there exists degenerate solutions when there is an extra light sterile neutrino.

\section{Results for hierarchy sensitivity}

To study the effect of these degeneracies on the hierarchy measurement in Fig. 3 we have plotted the hierarchy $\chi^{2}$ in the true $\theta_{23}$-true $\delta_{13}$ plane. From the figure we see that NOvA has good hierarchy sensitivity for $\delta_{13}=-90^{\circ}$ for the generation case. But for the $3+1$ case, the sensitivity depends on the true value of $\delta_{14}$. For $\delta_{14}=-90^{\circ}$ we see that the hierarchy sensitivity is lost for $\theta_{23}<43^{\circ}$ if the octant is unknown. However if the octant is known then the sensitivity coincides with the three generation case. For $\delta_{14}=0^{\circ}$, we note that the hierarchy sensitivity is lost if $\theta_{23}$ is less than $46^{\circ}$ for both the cases. However the most remarkable result is obtained if $\delta_{14}$ is $90^{\circ}$. In this case we see that there is a complete loss of hierarchy sensitivity at $2 \sigma$ for all true values of $\theta_{23}$. 


\section{Summary}

In this work we have studied the parameter degeneracy in neutrino oscillation in the presence of a light sterile neutrino in the $\mathrm{eV}$ scale for NOvA. In our work we have identified new degeneracies which are absent in the standard three generation case. Because of these there are unsolved degenerate region in the $3+1$ case. We also showed that the hierarchy sensitivity depends on the true values of $\theta_{14}$. If the observed hierarchy sensitivity of NOvA is less than the expected then this can be a hint of existence of sterile neutrinos. For more detail we refer to [10] on which this article is based upon.

\section{Acknowledgements}

The work of MG is partly supported by the "Grant-in-Aid for Scientific Research of the Ministry of Education, Science and Culture, Japan", under Grant No. 25105009. SG, ZM, PS and AGW acknowledge the support by the University of Adelaide and the Australian Research Council through the ARC Centre of Excellence for Particle Physics at the Terascale (CoEPP) (grant no. CE110001004).

\section{References}

[1] P. Adamson et al. [NOvA Collaboration], "Constraints on Oscillation Parameters from ve Appearance and $v_{\mu}$ Disappearance in NOvA," Phys. Rev. Lett. 118, no. 23, 231801 (2017) [arXiv:1703.03328 [hep-ex]].

[2] D. V. Forero, M. Tortola and J. W. F. Valle, "Neutrino oscillations refitted," Phys. Rev. D 90, no. 9, 093006 (2014) [arXiv:1405.7540 [hep-ph]].

[3] I. Esteban, M. C. Gonzalez-Garcia, M. Maltoni, I. Martinez-Soler and T. Schwetz, "Updated fit to three neutrino mixing: exploring the accelerator-reactor complementarity," JHEP 1701, 087 (2017) [arXiv:1611.01514 [hep-ph]].

[4] F. Capozzi, G. L. Fogli, E. Lisi, A. Marrone, D. Montanino and A. Palazzo, "Status of three-neutrino oscillation parameters, circa 2013,” Phys. Rev. D 89, 093018 (2014) [arXiv:1312.2878 [hep-ph]].

[5] K. N. Abazajian et al., "Light Sterile Neutrinos: A White Paper," arXiv:1204.5379 [hep-ph].

[6] N. Klop and A. Palazzo, "Imprints of CP violation induced by sterile neutrinos in T2K data," Phys. Rev. D 91, no. 7, 073017 (2015) [arXiv:1412.7524 [hep-ph]].

[7] J. Kopp, P. A. N. Machado, M. Maltoni and T. Schwetz, "Sterile Neutrino Oscillations: The Global Picture," JHEP 1305, 050 (2013) [arXiv:1303.3011 [hep-ph]].

[8] P. Huber, M. Lindner and W. Winter, "Simulation of long-baseline neutrino oscillation experiments with GLoBES (General Long Baseline Experiment Simulator)," Comput. Phys. Commun. 167, 195 (2005) [hep-ph/0407333].

[9] S. K. Agarwalla, S. S. Chatterjee and A. Palazzo, "Octant of $\theta_{23}$ in danger with a light sterile neutrino,” Phys. Rev. Lett. 118, no. 3, 031804 (2017) [arXiv:1605.04299 [hep-ph]].

[10] M. Ghosh, S. Gupta, Z. M. Matthews, P. Sharma and A. G. Williams, "Study of parameter degeneracy and hierarchy sensitivity of NOvA in presence of sterile neutrino," Phys. Rev. D 96, no. 7, 075018 (2017) [arXiv:1704.04771 [hep-ph]]. 